\title{
Conservative Management After a Single Fetal Death in a Dichorionic Diamniotic Twin Pregnancy and Fetus Papyraceus: A Case Report
}

\begin{abstract}
Authors:
*Rayra Amana Macêdo Maciel, ${ }^{1}$ Caio Ribeiro Vieira Leal, ${ }^{1}$ Marina

Fistarol, ${ }^{1}$ Sara de Pinho Cunha Paiva, ${ }^{1}$ Moisés Salgado Pedrosa, ${ }^{2}$ Maria

Luiza Peloso Maia²

1. Department of Gynecology and Obstetrics, Clinics Hospital, Federal University of Minas Gerais, Belo Horizonte, Brazil

2. Department of Pathology, Clinics Hospital, Federal University of Minas Gerais, Belo Horizonte, Brazil

*Correspondence to rayraamana@gmail.com

Disclosure: $\quad$ The authors have declared no conflicts of interest.

Received: $\quad 22.02 .21$

Accepted: $\quad 11.06 .21$

Keywords: $\quad$ Fetal death, fetus papyraceous, twin pregnancy.

Citation: $\quad$ EMJ Repro Health. 2021;7[1]:72-76.

Abstract

Multifetal pregnancies are estimated to represent 3.2\% of all pregnancies (80\% are dichorionic and $20 \%$ monochorionic) and are associated with a higher risk of perinatal morbidity and mortality relative to single pregnancies. The authors report a successful case of conservative management of a dichorionic diamniotic twin pregnancy after a single fetal death in the second trimester of pregnancy. The diagnosis was made in the $22^{\text {nd }}$ week of pregnancy and the pregnancy was followed up until delivery in the $39^{\text {th }}$ week. A healthy $(2.855 \mathrm{~kg}$ ) female infant was born and a dead fetus, approximately $20 \mathrm{~cm}$ in length and connected by the rudimentary umbilical cord to the small calcified placenta, was seen. The occurrence of a single fetal death is a relatively common event, which has implications for maternal and fetal outcomes. This diagnosis is relevant due to its potential effect on the survival of the other fetus and on possible maternal complications. In order to avoid complications and achieve the optimal maternal and neonatal outcomes, conservative prenatal follow-up should focus on careful monitoring and serial assessment of both fetal and maternal wellbeing. In gestational losses where the fetus is retained intrauterine for at least 10 weeks, there is the possibility of finding fetus papyraceus at the time of delivery. This is a rare event that results from incomplete reabsorption of the dead fetus, which is then compressed between the membranes and the uterine wall.
\end{abstract}

\section{INTRODUCTION}

Multifetal pregnancies are estimated to represent $3.2 \%$ of all pregnancies (80\% are dichorionic and $20 \%$ monochorionic) ${ }^{1}$ and are associated with a higher risk of perinatal morbidity and mortality when compared to single pregnancies. ${ }^{2-4}$ The

occurrence of a single fetal death is a relatively common event, occurring in approximately $6 \%$ of cases, 2,4-6 and has several implications for maternal and fetal outcomes, such as maternal disseminated intravascular coagulation, hypertensive disorders of pregnancy, and structural abnormalities in the surviving fetus 
(e.g., cerebral alterations, bilateral renal cortical necrosis, gastrointestinal tract atresia, and aplasia cutis). ${ }^{7}$ The extrinsic compression of the dead fetus by the remaining fetus can result in the appearance of an entity known as fetus papyraceous, a rare event that occurs in 0.018-0.02\% of multifetal pregnancies. .,9 $^{8,9}$

Herein, the authors report a successful case of conservative management of a dichorionic diamniotic twin pregnancy after a single fetal death in the second trimester of pregnancy.

\section{CASE REPORT}

A 36-year-old patient (gravida 2, para 1; one previous caesarean section) was referred for follow-up at the high-risk prenatal care unit of a tertiary referral hospital due to a dichorionic diamniotic twin pregnancy at 11 weeks of pregnancy (calculated by an obstetric ultrasound performed at the $8^{\text {th }}$ week). The patient was known to have epilepsy, controlled using phenobarbital (100 mg daily), but no other comorbidities were recorded. At the $13^{\text {th }}$ week of pregnancy, another obstetric ultrasound was performed, showing two gestational sacs of usual aspect, containing, in each one, an embryo with a visible heartbeat and nasal bone. In addition, nuchal translucency screening tests were in the appropriate percentiles for gestational age (Figures 1 and 2 ). At the $16^{\text {th }}$ week of pregnancy, the patient was clinically examined and both twins' hearts were auscultated. No abnormalities were found on the clinical examinations.

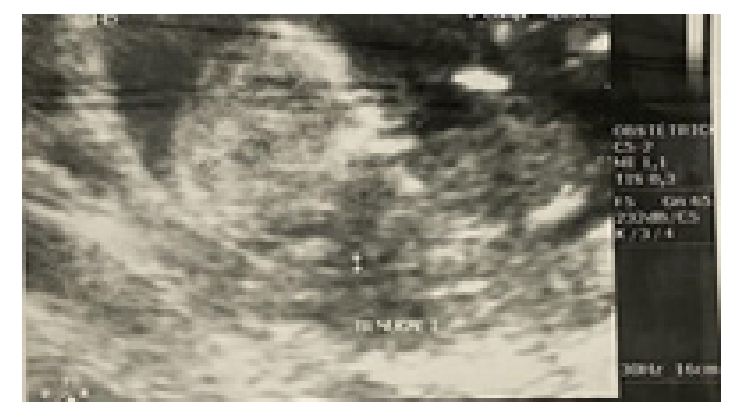

Figure 1: Ultrasound showing first twin with normal nuchal translucency screening and presence of nasal bone.

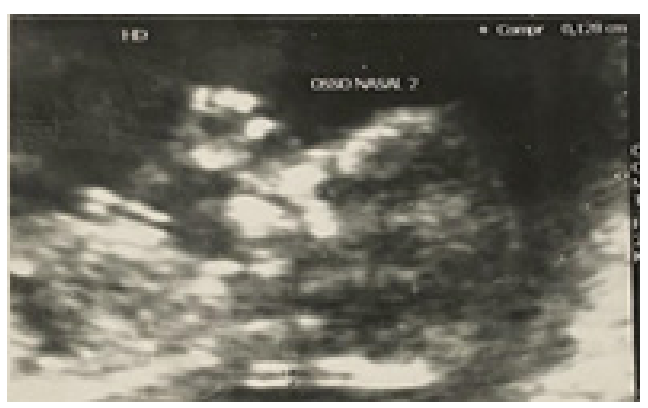

Figure 2: Ultrasound showing second twin with normal nuchal translucency screening and presence of nasal bone.

At the $22^{\text {nd }}$ week of pregnancy, a morphology ultrasound was performed and the death of one of the fetuses was diagnosed. The live fetus showed good vitality and no morphological changes were detected. The dead fetus was not described.
The pregnancy follow-up comprised a total of 12 prenatal visits and all tests recommended were performed, including three more ultrasound examinations, which were performed at 2324 weeks, 31-32 weeks, and 37-38 weeks. These consistently demonstrated a fetus with adequate growth and good vitality. There were 
no descriptions regarding the dead fetus in the ultrasounds.

At 39 weeks of gestational age, the patient was admitted to the maternity ward for caesarean section on request (according to the maternity protocol). Gestational hypertension was diagnosed on admission. The patient underwent an uneventful lower segment caesarean section. A healthy $(2.855 \mathrm{~kg})$ female infant was born, whose Apgar scores were 8 and 9 at the first and fifth minute of life, respectively. After extraction of the placenta, a dead fetus, approximately $20 \mathrm{~cm}$ in length and connected by the rudimentary umbilical cord to the small calcified placenta, was seen (Figure 3). The set of placentas and the mummified fetus were sent for anatomo-pathological examination. The mother and the newborn had no complications during hospitalisation and were discharged 72 hours after delivery.

The anatomo-pathological report showed a succenturiate placenta (an accessory lobe) and a marginally inserted umbilical cord containing three vessels. No other relevant histopathological lesions were observed.

Adhered to the membranes was an involuted placental disc of $15.0 \times 12.0 \times 0.3 \mathrm{~cm}$ with extensive areas of infarction/hyalinisation and calcification. In the same flask, there were a fetus papyraceus showing maceration Grade III, whose sex could not be morphologically determined, with an anthropometric gestational age of 13 weeks.

\section{DISCUSSION}

This report shows that conservative management can be successful for cases of single fetal death in a twin pregnancy during the second trimester. The prognosis of pregnancy after the death of one of the twins will depend primarily on the gestational age at the time of fetal death and chorionicity, regardless of amnionicity. If the loss occurs within the first trimester, the death of one of the fetuses does not appear to be associated with deleterious effects on the development of the survivor, especially in dichorionic diamniotic pregnancies. In this instance, patients may be asymptomatic or present with abdominal pain and mild genital bleeding. 2,8,9 However, single fetal death after 14 weeks, and especially after the $20^{\text {th }}$ week of pregnancy, is associated with adverse effects on the surviving fetus, with a higher risk of prematurity (spontaneous or iatrogenic), restricted intrauterine growth, neurological morbidity for the surviving fetus, pre-eclampsia, haemorrhage, and sepsis. ${ }^{2,4,5,8,10}$

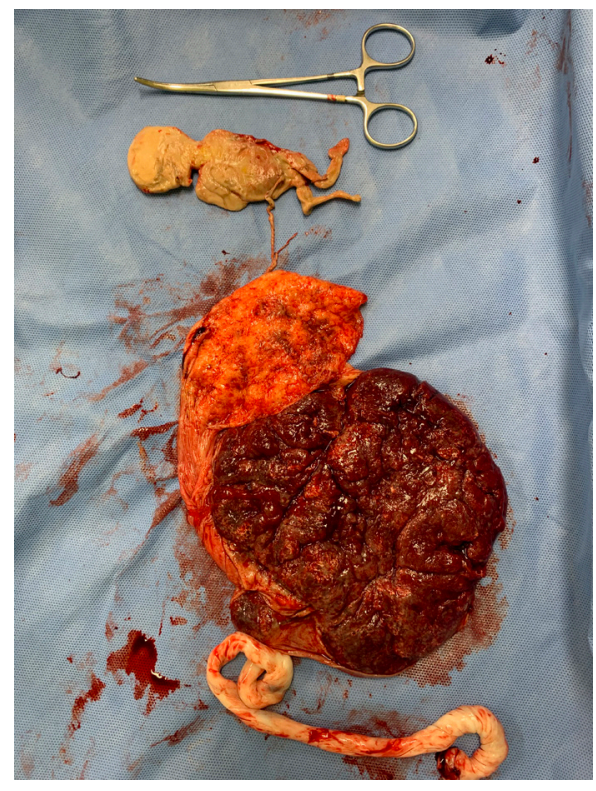

Figure 3: Macroscopically unchanged placenta adhered to calcified placental disc, which is connected by a rudimentary umbilical cord to a mummified fetus. 
The prognosis of the surviving fetus is worse in monochorionic pregnancies, regardless of amnionicity, due to mechanisms that are not yet well understood. However, it is most probably related to the presence of important vascular anastomoses that allow thrombotic substances released by the dead fetus to reach the circulation of the surviving fetus, causing hypotension, hypoperfusion, hypoxia, acidosis, exsanguination, severe anaemia, and generalised ischaemic injuries (particularly in the central nervous system of the surviving twin). ${ }^{9,1,1,2}$

In monochorionic twins, the risk of prematurity (most relevant between 28-33 weeks of pregnancy), neuropsychomotor disorders, postnatal cranial imaging abnormalities, and death of the surviving twin after single fetal death were estimated at $68 \%, 26 \%, 34 \%$, and $15 \%$, respectively, while in dichorionic twins the rates were estimated at $54 \%, 2 \%, 16 \%$, and $3 \%$, respectively. 10,12

Currently, there is no consensus regarding the follow-up or definition of the ideal gestational age for interruption of pregnancy in the event of the death of one of the fetuses in twin pregnancies. However, fetal death in the first trimester does not appear to be associated with adverse outcomes, a risk that increases from the second or third

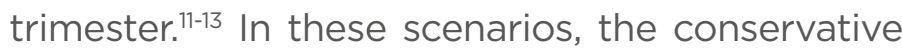
approach is advocated above all else when gestational loss of one of the fetuses occurs at a non-viable gestational age or is associated with extreme prematurity..$^{13}$ In the case of dichorionic pregnancies, pregnancy must be carried out for at least 38 weeks, whenever both maternal and fetal wellbeing are assured,, $, 0,1,13$ unless there is some other obstetric indication for termination of pregnancy. In the case of monochorionic pregnancies, conservative management is an option, especially prior to 34 weeks, because of the greater neonatal risks associated with prematurity. Antenatal corticosteroid therapy should be considered. ${ }^{13}$

In conservative management, follow-up should prioritise surveillance of fetal wellbeing through serial ultrasounds to monitor fetal growth and amniotic fluid volume. Doppler ultrasonographic measurement of the peak systolic velocity in the middle cerebral artery is a good parameter for monitoring fetal anaemia. This is very useful when assessing wellbeing due to the subsequent risk of exsanguination of the surviving fetus. . $3,14^{14}$ Persistent absent or reversed end-diastolic flow in umbilical artery Doppler has been associated with severe fetal deterioration, while intermittent absent or reversed end-diastolic flow has been reported to be associated with unexpected fetal demise. Normal umbilical artery Doppler pulsatility index carries the best prognosis. ${ }^{11,13,17}$

For maternal monitoring, coagulation blood tests are recommended. Moreover, special attention should be paid to blood pressure and proteinuria levels. This is due to the higher risk of hypertensive disorders associated with twin pregnancies, especially those in which one of the fetuses dies.13,15 Anti-Rho Ig should be administered to rhesus-negative patients. ${ }^{13}$ The determination of the mode of delivery must be based on obstetric criteria. ${ }^{11,13}$

Fetus papyraceous results from incomplete absorption of a dead fetus, retained inside the uterus for at least 10 weeks, which undergoes fluid loss and mechanical compression between the membranes and the uterine wall. The cause of death is generally unknown but it is often associated with chromosomal abnormalities, placental abnormalities such as improper insertion of the umbilical cord, or, in the case of monochorionic diamniotic pregnancies, twin-totwin transfusion syndrome. ${ }^{8}$ The cause of death of one of the fetuses in this case report was unknown. However, it is important to discuss one of the relevant comorbidities of the patient: epilepsy, which was treated with phenobarbital (100 mg daily). There are data in the literature showing that exposure to anti-epileptic drugs, especially phenobarbital, may be associated with unfavourable pregnancy outcomes. In a study conducted by Wen et al., ${ }^{16}$ 14,982 women were exposed to folic acid antagonists (either sulfamethoxazole-trimethoprim or phenobarbital). Overall, maternal exposure was associated with a greater risk of restricted fetal growth and fetal death. A prospective casecontrol cohort study conducted by Waters et al. ${ }^{17}$ also showed that women with epilepsy who were exposed to anti-epileptic drugs (carbamazepine, phenytoin, and phenobarbital) had a higher rate of fetal death and anomalies than the control population. In this instance, phenobarbital was associated with the highest relative risk for those outcomes. No data have been found in the literature concerning single fetal death in 
follow-up should focus on careful monitoring phenobarbital exposure.

and serial assessment of both fetal and maternal wellbeing.

\section{CONCLUSION}

The diagnosis of a single fetal death in multifetal pregnancies is relevant due to its potential effect on the survival of the other fetus and on possible maternal complications. In order to avoid complications and achieve the optimal maternal

In gestational losses where the fetus is retained intrauterine for at least 10 weeks, there is the possibility of finding fetus papyraceus at the time of delivery. This is a rare event that results from incomplete reabsorption of the dead fetus, which is compressed between the membranes and the uterine wall.

\section{References}

1. Martin JA et al. Births: final data for 2018. Natl Vital Stat Rep. 2019;68(13):1-47.

2. Cunningham FG et al. (eds.), Williams Obstetrics (2014) 24 ${ }^{\text {th }}$ edition, New York: McGraw-Hill Education.

3. Chen FJ et al. [Twin pregnancy complicated by one intrauterine fetal death. Report of a case and review of the literature]. Ginecol Obstet Mex 1995;63:352-5. (In Spanish).

4. Buonacorso R et al. [Twin pregnancy with death of one fetus: case report] Arq Med Hosp Fac Cienc Med Santa Casa São Paulo. 2006:51(3):88-91. (In Portuguese).

5. Mackie FL et al. Prognosis of the co-twin following spontaneous single intrauterine fetal death in twin pregnancies: a systematic review and meta-analysis. BJOG. 2019;126(5):569-78.

6. Pharoah PO, Adi Y. Consequences of in-utero death in a twin pregnancy. Lancet. 2000;355(9215):1597-602.
7. Woo $\mathrm{HH}$ et al. Single foetal death in twin pregnancies: review of the maternal and neonatal outcomes and management. Hong Kong Med J. 2000;6(3):293-300.

8. Gadre S, Gangatirkar R. Fetus papyraceous in monochorionic diamniotic twins. J Obstet Gynaecol India. 2019;69(Suppl 1):40-3.

9. Berceanu $\mathrm{C}$ et al. Morphological and ultrasound findings in multiple pregnancy placentation. Rom J Morphol Embryol. 2018;59(2):435-53.

10. Hillman SC et al. Co-twin prognosis after single fetal death: a systematic review and meta-analysis. Obstet Gynecol. 2011;118(4):928-40.

11. Vale-Fernandes E et al. Single fetal death in monochorionic twin pregnancy: co-twin prognosis and neonatal outcome. Acta Med Port. 2017;30(2);148-51.

12. Ong SSC et al. Prognosis for the co-twin following single-twin death: a systematic review. BJOG.
2006;113(9):992-8

13. Shek NWM et al. Single-twin demise: pregnancy outcome. Best Pract Res Clin Obstet Gynaecol. 2014;28(2):249 63.

14. Senat MV et al. The value of middle cerebral artery peak systolic velocity in the diagnosis of fetal anemia after intrauterine death of one monochorionic twin. Am J Obstet Gynecol. 2003;189(5):1320-4.

15. Dahiya P, Bains R. Conservative management of fetus papyraceus: a report of two cases. Oman Med J. 2014;29(2):132-4

16. Wen SW et al. Maternal exposure to folic acid antagonists and placentamediated adverse pregnancy outcomes. CMAJ. 2008;179(12):1263-

17. Waters $\mathrm{CH}$ et al. Outcomes of pregnancy associated with antiepileptic drugs. Arch Neurol. 1994;51(3):250-3. 